\title{
À propos des benchmarks en géotechnique
}

\section{Philippe Mestat - Yvon Riou*}

\author{
LCPC, 58 boulevard Lefebvre, F-75732 Paris cedex 15 \\ mestat@lcpc.fr \\ * Ecole Centrale de Nantes, BP 92101, F-44321 Nantes cedex 03 \\ Yvon.Riou@ec-nantes.fr
}

RESUME. Cet article aborde deux aspects de la vérification et de la validation des logiciels industriels d'éléments finis spécialisés dans le domaine de la géotechnique : la démarche de tests et la valeur d'exemple des cas-tests en vraie grandeur (benchmarks). D'une part, il traite des plates-formes de tests qu'il convient de mettre en place pour valider les codes de calcul et des problèmes particuliers rencontrés avec les essais sur ouvrage en vraie grandeur (ou benchmarks). Notamment, la difficulté de réaliser des calculs prévisionnels est analysée en termes de facteurs d'incertitudes (mesures et hypothèses de modélisation), de limitations des modèles et de procédures de détermination des paramètres. D'autre part, on a essayé de tirer de ces confrontations calculs-mesures sur ouvrages-types des enseignements et des critères de validation pour de futures modélisations. Les benchmarks apparaissent alors à la fois comme un outil de validation des modèles numériques et comme un outil d'apprentissage de la modélisation appliquée aux ouvrages du génie civil.

ABSTRACT. This paper deals with the process of checking and validating commercial finite element codes in the field of geotechnics, and specially the need for validation and learning exercises. We propose, herein, some general informations about benchmark-workshops that need to be organized in order to be ensure the validity of softwares or to assess the limits of computational codes. In particular, practical and numerical modelling difficulties inherent to full scale geotechnical structures tests are examined : for example no assurance on measure, the user's choice for initial stress state, the limitation of the model, the choice of parameters for the constitutive law, etc. On the other hand, for future benchmarks, some instructions and validity criteria are proposed from various observed - calculated case histories of geotechnical structures.

Mors-CLES. Méthode des éléments finis, modélisation, géotechnique, benchmark, test, validation, vérification, qualification, qualité.

KEYWORDs. Finite element method, modelling, geotechnics, benchmark, test, validation, control, qualification, quality. 
"Un test est un protocole expérimental très précis et reproductible qui doit provoquer un ensemble de réponses qui montrent que la transformation opérée par le programme est correcte. » Jacques Printz (Le génie logiciel)

\section{Introduction}

Les responsables de bureaux d'études et de contrôle sont souvent méfiants devant les résultats des calculs par éléments finis et la précision des chiffres qui leur sont présentés. Les principales raisons de cette méfiance semblent être les suivantes :

- la difficulté de comparer avec les résultats des méthodes traditionnelles, qui est due notamment à des fondements théoriques différents ;

- le fait que « n'importe qui » est aujourd'hui capable de faire un tel calcul grâce aux progrès réalisés en matière de convivialité des logiciels et de visualisation des résultats ;

- la masse d'informations importante fournie par le moindre calcul par éléments finis (déplacements, contraintes, rotations, pressions interstitielles, moments et efforts, mécanisme de déformation et de rupture, etc.) et le risque d'une mauvaise interprétation ;

- le manque de validation des modèles par rapport aux ouvrages réels, de publications de synthèse et d'exemples de référence qui puissent inspirer les utilisateurs relativement inexpérimentés ;

- l'absence d'une démarche d'Assurance-Qualité formalisée qui pourrait garantir la fiabilité des logiciels et leur bonne utilisation dans le domaine de la géotechnique. Une telle démarche a été initiée dans d'autres domaines, comme celui du calcul des structures ou des barrages. On peut citer les travaux de la Commission de Validation des Progiciels de Calcul de Structures (créée en 1987 au sein de la Société Française des Mécaniciens), de la Commission Internationale des Grands Barrages (CIGB, 1994) et ceux menés par la NAFEMS (National Agency for Finite Element Methods and Standards en Grande-Bretagne).

Derrière ces arguments apparaissent les points essentiels de l'Assurance-Qualité concernant les codes de calcul par éléments finis :

- la « vérification " qui consiste à vérifier que le programme fait bien ce que l'on attend qu'il fasse. Les résultats fournis doivent être en accord avec des solutions connues. Il n'y a pas de bogues et les algorithmes sont bons ;

- la « validation» qui procure l'assurance que le logiciel fournit une réponse correcte au problème posé. Les résultats sont corrects et cohérents avec les hypothèses de calcul. On parlera de validation du logiciel. La validation doit également montrer 
que le logiciel donne de bons résultats sur des cas réels. On pariera alors de validation du modèle (réalisé avec le logiciel) ;

- la « qualification » des utilisateurs, qui assure que l'ingénieur chargé du calcul est apte à réaliser le modèle demandé ;

- la " justification ", qui permet de démontrer que le modèle réalisé est capable de décrire la réalité du fonctionnement de l'ouvrage étudié. Un utilisateur qualifié doit savoir justifier son modèle avec pertinence. Cette opération intervient dans la démarche de validation du modèle et devrait être considérée dans toute présentation du modèle (note de calcul, rapport, article, communication, etc.).

Pour vaincre les réticences des responsables d'études et sensibiliser les maîtres d'ouvrage, le moyen le plus efficace consiste à pratiquer des tests avec deux objectifs principaux :

- valider les logiciels en condition normale d'emploi (la validation doit être globale et concerner autant que possible l'ensemble des potentialités du logiciel). Il s'agit aussi de privilégier les tests de type industriel, c'est-à-dire ceux qui nécessitent une confrontation des résultats avec des mesures réalisées sur des ouvrages-types ;

- former les utilisateurs par l'exemple, puis les qualifier par des tests d'aptitude. Les tests considérés ne font pas forcément référence à des expérimentations ou à des cas complexes, mais ils doivent s'en inspirer pour que la formation soit profitable.

De nombreuses recherches ont été consacrées à la validation des codes de calcul par rapport à des essais sur modèles physiques. Même si les modèles numériques sont encore imparfaits vis-à-vis de la complexité de la réalité géotechnique, il est aujourd'hui intéressant et utile de tirer des enseignements de ces multiples confrontations entre calculs et mesures et de dégager des recommandations pour de futures modélisations d'ouvrages (choix de modèles et principes de contrôle des résultats) afin de tendre vers le second objectif.

\section{Tester les logiciels d'éléments finis : de la vérification à la validation}

La vérification et la validation constituent une préoccupation permanente des concepteurs et une légitime revendication des utilisateurs. Malheureusement, ce sont des opérations longues et délicates à mener. Toutes les deux nécessitent de recourir à des tests et à des confrontations avec des informations extérieures au logiciel. Plusieurs catégories de tests peuvent être ainsi distinguées. 


\subsection{Plate-forme de tests pour la vérification}

Pour un logiciel général de calcul, il est quasiment impossible de contrôler toutes les combinaisons d'emploi disponibles. Face au nombre de tests théoriquement nécessaire, le problème est donc de sélectionner une plate-forme de «bons " tests, les plus pertinents et les plus efficaces. Pour cela, il est nécessaire d'élaborer une stratégie fondée sur un ensemble de tests des plus simples aux plus complexes :

- les tests unitaires pour vérifier les résultats au niveau des sous-programmes ;

- les tests d'intégration pour tester un ensemble de sous-programmes qui viennent d'être réunis dans le logiciel ;

- les tests de non-régression pour s'assurer que la programmation n'est pas altérée par des modifications du code (corrections, rapatriement de source dans une version diffusée) ou de l'environnement informatique ;

- les tests de robustesse pour estimer la réponse du logiciel à des données erronées (par exemple : des valeurs non physiques pour les paramètres) ;

- les tests élémentaires pour contrôler les résultats d'applications simples avec des solutions théoriques connues (elles sont relativement rares dans le domaine de la géotechnique). Ces tests doivent aussi permettre de tester des combinaisons de types d'analyse (élastique, élastoplastique, thermique, dynamique, etc.), de géométries, d'éléments finis, de chargements, de conditions aux limites, etc.

Les tests unitaires et élémentaires servent à démontrer les performances des algorithmes et l'absence d'erreurs dans le code (autant que faire se peut) par un contrôle de l'exactitude des résultats et à fabriquer une bibliothèque de tests automatiques afin de vérifier régulièrement que la programmation n'a pas été altérée. Ce travail est indispensable en phase de développement, de changement de version ou encore lorsque l'utilisateur acquiert un nouvel ordinateur. Les tests d'intégration et de non-régression concernent surtout les concepteurs et sont effectués au cours du développement ou de la maintenance.

\subsection{Plate-forme de tests pour la validation}

La validation nécessite des tests particuliers qui doivent servir à prouver la validité du logiciel et du modèle, c'est-à-dire l'aptitude à résoudre de manière satisfaisante un problème de géotechnique. Ils sont élaborés à partir de scénarios aussi représentatifs que possible des besoins les plus fréquents des utilisateurs en matière de modélisation. L'emploi d'une méthode de type " cause - effet " peut faciliter l'analyse et déterminer la couverture de tests : les sorties du diagramme cause-effet 
seront autant de tests à effectuer. D'une manière générale, on distingue deux types de tests :

- les tests semi-industriels destinés à vérifier la validité des domaines d'emploi du logiciel. Il peut s'agir de comparer les résultats avec des solutions de référence (calculées par d'autres logiciels) ou avec des mesures sur des modèles physiques (centrifugés ou non). Ces tests sont réalisés avec une certaine maîtrise de la nature des matériaux considérés (homogénéité notamment) qui rend plus facile la modélisation des essais, mais qui éloigne quelque peu de la réalité des projets ;

- les tests industriels voués à démontrer l'aptitude du logiciel à modéliser des ouvrages dans des conditions proches de celles du projet. La validation provient d'un bon accord entre les résultats numériques et les mesures réalisées sur des ouvrages en vraie grandeur. La confrontation concerne à la fois le logiciel, la justification des hypothèses du modèle et la manière de les mettre en oeuvre.Ces tests constituent les véritables et seuls tests de validation au sens strict.

\subsection{Problématique des * tests industriels » en géotechnique}

Les tests industriels ou « benchmarks » sont l'outil incontournable pour la validation d'un logiciel en condition normale d'utilisation, car il n'existe pas de solution théorique pour le calcul des ouvrages (à moins de considérer des ouvrages très schématisés et un comportement du sol élastique linéaire). Malheureusement, si la validation en vraie grandeur est la seule véritablement pertinente, elle est complexe car on en vient à tester simultanément le logiciel et la manière dont un utilisateur peut s'en servir.

La complexité de la comparaison tient également aux incertitudes liées au modèle qu'il faut mettre en oeuvre et au soin qu'il faut apporter à l'expérimentation in situ. Plusieurs phénomènes peuvent intervenir simultanément et compliquer le modèle ou l'interprétation des mesures : c'est le cas notamment des phénomènes d'interactions sol-structures et de l'effet de l'eau dans les sols. Il existe donc de nombreux facteurs d'incertitudes susceptibles de perturber la validation sur ouvrages réels (tableau 1). À cause de ceux-ci, les expérimentations de référence utilisables à des fins de validation demeurent très rares. La complexité de la construction, l'influence du phasage des travaux (battage, fonçage, etc.), les conditions hydrauliques, les conditions aux limites et initiales ou le comportement véritablement tridimensionnel (tirants, dimensions finies des ouvrages) sont autant de problèmes qui empêchent de maîtriser les conditions expérimentales et rendent délicate l'élaboration du modèle numérique pour décrire le comportement mesuré.

Une bonne validation passe donc par de bonnes mesures, fiables et précises. Si le calcul numérique s'est développé très rapidement, il n'en est pas de même des 
techniques de mesure et des types d'essai de sol pratiqués couramment in situ et en laboratoire. Des progrès dans ces domaines seraient les bienvenus de manière à améliorer la comparaison entre les résultats et les mesures (déplacements, pressions interstitielles, contraintes et efforts appliqués par le sol aux structures).

\begin{tabular}{|l|l|}
\hline \multicolumn{1}{|c|}{ Incertitudes relatives au modèle } & Incertitudes relatives à une expérience \\
\hline - Représentativité de la théorie & - Trouver un sol suffisamment \\
- Hypothèse du milieu continu et de & homogène pour éviter une trop grande \\
matériaux saturés & variabilité des propriétés des sols \\
- Simplification de la géométrie & - Réalisation d'essais in situ (nombre et \\
(déformation plane ou axisymétrique) & positions) pour la reconnaissance \\
- Hypothèses sur les discontinuités & - Conditions aux limites (mécanique, \\
- Type d'éléments et finesse du maillage & variation de la nappe phréatique) \\
- Choix des lois de comportement & - Conditions initiales (état de \\
- Détermination des paramètres (à partir & contraintes, état de saturation du sol) \\
d'essais en place et en laboratoire) & - Précision des mesures \\
- Variabilité des paramètres & - Positions, densité et fiabilité des \\
- Etat initial mécanique et hydraulique & appareils de mesure \\
- Comportement d'interfaces & - Représentativité des échantillons \\
- Chargement et phasage des travaux & prélevés \\
- Algorithmes de résolution en & - Types d'essais à réaliser en laboratoire \\
comportement non linéaire ou couplé & (chemins de contraintes) \\
& - Déroulement réel des travaux \\
\hline
\end{tabular}

Tableau 1. Facteurs d'incertitude dans les modélisations en géotechnique

\subsection{Comparaison des comportements calculé et observé}

En dépit des incertitudes évoquées précédemment et que les expérimentateurs tentent de maîtriser, la comparaison entre les comportements observé et calculé est possible à partir du moment où on a bien repéré les aspects caractéristiques du fonctionnement de l'ouvrage étudié et donc la position optimale pour les instruments de mesure : savoir ce qui doit être mesuré et où on le mesure. Un cahier des charges précis et rigoureux de l'instrumentation doit accompagner l'expérimentation. Ce cahier des charges peut aussi s'appuyer sur des modélisations préalables de l'expérimentation, par exemple à des fins de dimensionnement. L'interprétation des mesures permet ensuite d'obtenir les quantités à comparer. Celles-ci dépendent du type d'ouvrage analysé et des méthodes traditionnelles de calcul de la mécanique des sols. Dans le tableau 2, une liste de comparaisons essentielles est proposée pour chaque type d'ouvrage de géotechnique. 


\begin{tabular}{|l|l|}
\hline \multicolumn{1}{|c|}{ Type d'ouvrage } & \multicolumn{1}{|c|}{ Quantités à comparer (calculs-mesures) } \\
\hline $\begin{array}{l}\text { Fondations } \\
\text { superficielles }\end{array}$ & $\begin{array}{l}\text { Courbe charge-tassement au centre de la fondation ; rotation de } \\
\text { la fondation ; capacité portante ; tassement différé dû au fluage } \\
\text { pour les sols fins. }\end{array}$ \\
\hline $\begin{array}{l}\text { Fondations } \\
\text { profondes }\end{array}$ & $\begin{array}{l}\text { Courbe charge-tassement au centre de la fondation ; charge en } \\
\text { pointe et charge de frottement latéral ; capacité portante. }\end{array}$ \\
\hline $\begin{array}{l}\text { Ouvrages de } \\
\text { soutènement }\end{array}$ & $\begin{array}{l}\text { Déplacements du sol en surface et en fond de fouille ; } \\
\text { déformée du soutènement ; efforts et moments dans les } \\
\text { structures (butons, tirants, tension d'ancrage) ; pressions des } \\
\text { terres sur le soutènement ; variation des pressions } \\
\text { interstitielles ; profondeur limite du fond de fouille. }\end{array}$ \\
\hline Remblais & $\begin{array}{l}\text { Déplacements du sol en surface, sur l'axe et près du pied du } \\
\text { remblai ; variations des pressions interstitielles pendant et après } \\
\text { la construction; contraintes dans le remblai (fissuration) et } \\
\text { hauteur limite du remblai ; débit dans les drains. }\end{array}$ \\
\hline $\begin{array}{l}\text { Ouvrages en sols } \\
\text { renforcés }\end{array}$ & $\begin{array}{l}\text { Déplacements du mur et du sol en surface ; déformée des } \\
\text { structures ; efforts et moments dans les renforcements. }\end{array}$ \\
\hline $\begin{array}{l}\text { Ouvrages } \\
\text { souterrains }\end{array}$ & $\begin{array}{l}\text { Tassements en surface, déplacements horizontaux et verticaux } \\
\text { dans le massif encaissant (convergence de la galerie) ; efforts } \\
\text { et moments dans le revêtement. Mouvements instantanés et } \\
\text { différés pour certains milieux. }\end{array}$ \\
\hline $\begin{array}{l}\text { Pentes et versants } \\
\text { naturels }\end{array}$ & $\begin{array}{l}\text { Mouvements de la masse du sol ; déplacements suivant une } \\
\text { verticale et déplacements en crête ; surface de glissement. }\end{array}$ \\
\hline
\end{tabular}

Tableau 2. Caractéristiques pour la comparaison des résultats calculés et mesurés

Le tableau 3 précise les deux schémas devant lesquels l'ingénieur ou le chercheur peut se retrouver lorsqu'il compare ses résultats à des mesures. La conclusion de la confrontation n'est pas toujours évidente et elle peut conduire à une validation du modèle ou à une défiance vis-à-vis des mesures, ou laisser dans l'expectative et conduire à rechercher une autre expérience mieux maîtrisée.

La constitution d'une plate-forme de tests est un travail fondamental, long et coûteux, notamment pour sa maintenance et sa documentation ; chaque test devant être décrit avec soin. La régularité des passages (pour un logiciel en évolution) augmente également les coûts. Certains experts ont estimé que si l'activité de programmation compte pour environ 15 à $20 \%$ dans l'effort de développement d'un logiciel, la vérification et la validation peuvent en représenter de l'ordre de $40 \%$ (Gaudel et al., 1998). Le pourcentage restant est accaparé par la documentation. La maîtrise du processus de test est le garant de la qualité d'un logiciel. 


\begin{tabular}{|l|l|}
\hline \multicolumn{1}{|c|}{ Comparaison observations-calculs } & \multicolumn{1}{c|}{ Conclusions } \\
\hline $\begin{array}{l}\text { Comportement calculé différent de } \\
\text { celui qui a été observé et mesuré. Par } \\
\text { exemple : différence de signe sur les } \\
\text { déplacements ou les contraintes, } \\
\text { rapport de déplacements supérieur à 2. }\end{array}$ & $\begin{array}{l}\text { Erreurs dans le modèle (conception, } \\
\text { élaboration, données), incertitudes sur les } \\
\text { mesures ou instruments de mesure } \\
\text { défaillants. }\end{array}$ \\
\hline $\begin{array}{l}\text { Comportement calculé qualitativement } \\
\text { proche des observations. Par exemple, } \\
\text { les mouvements de sol et la pression } \\
\text { des terres sur l'ouvrage sont réalistes. }\end{array}$ & $\begin{array}{l}\text { On peut comparer les calculs aux } \\
\text { mesures, améliorer le modèle de calcul ou } \\
\text { encore pratiquer une analyse inverse. Il } \\
\text { s'agit de la situation la plus courante. }\end{array}$ \\
\hline
\end{tabular}

Tableau 3. Calcul prévisionnel et comportement observé

\section{Les concours de prévision du comportement des ouvrages}

\subsection{Types de calculs prévisionnels}

Lambe (1973) a distingué plusieurs types de calcul prévisionnel en fonction des données disponibles (tableau 4):

- une prévision de classe $\mathrm{A}$ est réalisée avant la construction de l'ouvrage et est fondée sur les données connues à cette période. C'est la situation habituelle de l'ingénieur qui doit étudier un projet d'ouvrage ;

- une prévision de classe $B$ est effectuée pendant la construction et peut bénéficier des premières mesures réalisées sur l'ouvrage. Si aucun calage n'est opéré, la prévision est dite de classe B. En revanche, si un calage est fait sur les premières mesures, la prévision est de classe $\mathrm{B} 1$;

- une prévision de classe $C$ est calculée après la fin de la construction. Si les mesures ne sont pas utilisées pour un recalage, la prévision est de classe $\mathrm{C}$. Dans le cas contraire, elle est dite de classe $\mathrm{Cl}$.

\begin{tabular}{|c|c|c|}
\hline Classe de prévision & Moment de la prévision & $\begin{array}{c}\text { Disponibilité des mesures } \\
\text { pour les modélisateurs }\end{array}$ \\
\hline A & avant l'essai & - \\
\hline B & pendant l'essai & non connues \\
\hline B1 & pendant l'essai & connues \\
\hline C & après l'essai & non connues \\
\hline Cl & après l'essai & connues \\
\hline
\end{tabular}

Tableau 4. Classement des calculs prévisionnels d'après Lambe (1973) 
En termes de validation et de démonstration de la capacité d'un logiciel à prévoir le comportement des ouvrages, les prévisions de classe A sont les plus probantes, mais ce sont aussi les plus rares. Cette rareté s'explique par le coût des expérimentations, leur délai (pas souvent conciliable avec les contraintes économiques d'un chantier) et l'effort d'interprétation qu'il faut mettre en oeuvre. Toutefois, la multiplication des grands projets innovants et des travaux en milieu urbain a permis d'instrumenter de nombreux ouvrages avec succès. Par ailleurs, depuis une quinzaine d'années, quelques ouvrages-types ont été spécialement construits, de par le monde, pour réaliser des expérimentations en vraie grandeur avec le souci de maîtriser le plus possible les facteurs d'incertitude. Autour de ces expérimentations, des concours de prévisions de classe A ont été organisés.

Si les prévisions de classe B sont également intéressantes, elles sont moins démonstratives. Elles gardent cependant tout leur intérêt pour suivre la vie de l'ouvrage et prévoir à court ou long terme son évolution. Le modèle est alors alimenté sur la base des informations recueillies au cours des différentes phases de construction de l'ouvrage et lors de son fonctionnement. À chaque perfectionnement du modèle (soit des paramètres mieux caractérisés, soit un changement de modèle conceptuel), correspond un recalcul de l'ouvrage depuis le début de sa construction (modèle accompagnateur de projet, (Tardieu et Ozanam, 1997)). En revanche, la publication de prévisions de classe $C$ n'apporte pas grand chose du point de vue de la validation. Toutefois, au niveau de la démarche de modélisation des ouvrages, certaines de ces études devraient inspirer l'élaboration des modèles en conditions de projet.

\subsection{Concours de prévisions de classe A}

Régulièrement, des concours de prévisions se déroulent dans le cadre de groupes de travail internationaux. Cette pratique permet d'une part de confronter des prévisions en aveugle (de classe $A$ ) et, d'autre part, de constituer des banques de données expérimentales détaillées sur le fonctionnement des ouvrages. À notre connaissance, les principaux concours de prévisions organisés ces dernières années (tests semiindustriels et industriels) sont les suivants.

Pour les fondations superficielles, le GRECO-Géomatériaux (1985-1992) a organisé un concours de prévisions sur des modèles de fondations circulaires centrifugés (Cambou et al., 1995). Par ailleurs, la Federal HighWay Administration (USA) a réalisé cinq essais de fondations carrées, de largeur variant de 1 à $5 \mathrm{~m}$, soumises à un chargement monotone (Gibbens et Briaud, 1994), qui ont également donné lieu à des prévisions des courbes charges-tassements. 
Pour les barrages, la Commission Internationale des Grands Barrages (CIGB) a organisé trois benchmarks internationaux dont notamment celui consacré au barrage en remblai d'El Infernillo (CIGB, 1994). La comparaison a porté sur des analyses statique et dynamique de la stabilité du barrage.

Pour les remblais sur sols compressibles, quatre concours ont été organisés successivement par le MIT (remblai de Boston, 1975), par la Division of Building research du Conseil National de la Recherche du Canada (remblai de Gloucester, près d'Ottawa, 1987), par la Direction des Routes de Malaisie (remblai de Muar Flats, 1989), par la Direction des Routes de Finlande (remblai d'Haarajoki, 1997). Les comparaisons demandées concernaient l'évolution dans le temps des déplacements et des pressions interstitielles (Lépidas et Magnan, 1990 ; Magnan, 1992 ; Aalto et al., 1998).

Pour les ouvrages souterrains, plusieurs concours ont été proposés dans le cadre de l'étude des stockages de déchets. On peut évoquer en particulier les projets européens COSA I et II pour le stockage dans des cavités de sel, auxquels ont participé dix laboratoires et centres de recherche (Piper et Knowles, 1988), et le projet européen mené sur le site de Mol en Belgique pour le stockage dans un massif argileux (argile de Boom) (de Bruyn et al., 1989). La confrontation était concentrée sur les prévisions de comportement au cours du temps (à court et moyen terme) : évolution des déplacements et des pressions interstitielles sur quelques années. On peut également citer des comparaisons avec des résultats obtenus sur des modèles centrifugés (Cambou et al., 1995).

Pour les ouvrages de soutènement, on peut citer le rideau de palplanches butonné de Hochstetten (Allemagne). Organisée par l'Université de Karlsruhe et le Dutch Center for Civil Engineering Research and Codes (CUR), la confrontation portait sur des résultats mesurés à différentes étapes du creusement de l'excavation et de la pose du soutènement (buton) (von Wolffersdorff, 1994): déplacements du rideau et tassements en surface, pression des terres sur le soutènement, moments fléchissants.

Malgré toutes les précautions prises (sol relativement homogène, chargement monotone, etc.), la confrontation des résultats numériques aux mesures montre que les calculs prévisionnels (de classe A) restent délicats à mener à cause des insuffisances des lois de comportement des sols, de la caractérisation de leurs paramètres et de celles des lois d'interface. Une conclusion importante se dégage de ces benchmarks : les analyses fondées sur des lois de comportement de sol de type élasto-plastique parfait permettent de décrire de manière relativement satisfaisante certains aspects du fonctionnement des ouvrages et du sol environnant, mais d'autres aspects sont mal représentés ou ignorés par le modèle (par exemple : la déformée d'un soutènement peut avoir une allure correcte alors que les tassements en surface ne correspondent pas aux observations). Ces analyses conduisent aussi fréquemment 
à des valeurs de déplacements supérieures à ce qui est mesuré. Cela est dû essentiellement aux valeurs des paramètres mécaniques qui sont estimées à partir d'essais en laboratoire réalisés sur des éprouvettes de sol remanié.

Pour leur part, les prévisions de classe A, effectuées avec des lois de comportement avancées pour le sol (élastoplasticité avec écrouissage), sont délicates à mener car les types d'essais de laboratoire et in situ ne sont pas toujours ceux souhaités et leur nombre peut être insuffisant pour déterminer avec précision l'ensemble des paramètres. Les résultats obtenus peuvent donc être aléatoires à cause du manque de données. En revanche, la réalisation de prévisions de classe $\mathrm{B} 1$ ou $\mathrm{Cl}$ avec des lois avancées conduit, après quelques calages, à une bonne description du fonctionnement général de l'ouvrage étudié, pourvu que les comportements d'interface et le couplage hydromécanique soient bien pris en compte. Toutefois, les modèles actuels les plus sophistiqués trouvent vite leurs limitations face à la complexité de la réalité, c'est notamment le cas lorsque les massifs de sol sont renforcés par des inclusions ou partiellement saturés.

De nombreuses causes peuvent donc altérer les jugements formulés au cours des comparaisons, ce qui incline à une certaine prudence dans les conclusions. Des courbes différant d'une dizaine d'unités ( $\mathrm{cm}$ ou $\mathrm{kPa}$ ) peuvent être en fait en excellent accord alors que des courbes superposées peuvent être le fruit du hasard. Malgré ces difficultés, il convient de persévérer car l'expérimentation est incontournable et demeure la seule possibilité de vérifier ou de rejeter les hypothèses de calcul, et donc de parfaire les modèles d'ouvrage.

\section{De l'intérêt des benchmarks pour la justification des modèles}

L'un des principaux enseignements des benchmarks est qu'ils doivent aider à dégager des critères de justification des modèles et permettre d'apprendre à modéliser les ouvrages. En effet, l'effort accompli pour réaliser des calculs prévisionnels ou pour caler un modèle conduit à

- réfléchir sur le mode de fonctionnement prédominant de l'ouvrage et de son environnement (interaction sol-structures, effet de l'eau, fluage, etc.) ;

- savoir simplifier à bon escient, choisir les bonnes hypothèses de calcul (détermination des paramètres) et bien utiliser les moyens informatiques et théoriques actuels.

La définition des critères de justification doit être facilitée par les études de synthèse réalisées sur le comportement observé d'un grand nombre d'ouvrages du même type et par les résultats des benchmarks. Ces critères, joints au contrôle de la pertinence des concepts fondamentaux du modèle (milieux continus, élastoplasticité, 
consolidation, etc.), peuvent servir de base pour la justification des modèles en conditions de projet. Les critères sont directement liés à l'évolution des caractéristiques regroupées dans le tableau 2 en fonction des chargements appliqués, représentant les différentes étapes de la construction et les charges de service.

Par exemple, dans le cas des remblais sur sol compressible, quatre critères de validation ont pu être établis (d'après Magnan, 1986) :

- lors de la construction, la pression interstitielle croît d'abord moins vite, puis à peu près aussi vite que la charge appliquée par le remblai, et elle augmente plus vite que la charge au voisinage de la rupture ;

- la distribution des déplacements horizontaux du sol sous les talus des remblais garde une forme constante au cours du temps ;

- le déplacement horizontal maximal sous le pied du talus augmente proportionnellement au tassement de l'axe du remblai, avec un coefficient de proportionnalité d'abord faible, puis voisin de l'uité pendant la construction, puis de nouveau faible après la fin de la construction ;

- les déplacements horizontaux évoluent vers l'extérieur du remblai.

Des critères similaires peuvent être mis en évidence pour les ouvrages souterrains, les soutènements ou les fondations. Ces critères, ajoutés aux divers contrôles et vérifications évoqués dans l'article de Mestat et al. (1999), devraient permettre de conclure à la « validation » d'un modèle d'ouvrage en condition de projet.

Un bon modèle de fonctionnement devrait être capable de prévoir simultanément tous les aspects importants de la réponse d'un ouvrage et de son environnement aux sollicitations qui lui sont imposées. Malheureusement, il est très rare qu'un modèle donne d'excellents résultats pour l'ensemble des critères. Les comparaisons sont alors globalement satisfaisantes, avec des concordances plus ou moins bonnes, selon les régions du maillage concernées. Il est aussi courant que les résultats diffèrent de plus en plus à mesure que l'intensité du chargement croît ou qu'un phénomène physique, qui n'a pas été considéré dans la modélisation, devient prépondérant (par exemple : l'interface entre les matériaux, l'écoulement de l'eau, etc.).

La transmission des connaissances acquises au cours des tests et des benchmarks est donc un enjeu important pour l'Assurance Qualité des calculs par éléments finis et une bonne pratique de la modélisation. Cela constitue d'ailleurs un des objectifs du Pôle d'expertise sur les éléments finis en génie civil de l'Association Française pour le Génie Civil (Prat et al., 1995 ; 1997). 


\section{Cas-tests : de nouveaux enjeux pour les utilisateurs et les concepteurs ?}

Pour les codes de type commercial, l'enjeu actuel des cas-tests n'est plus de réaliser des comparaisons avec des solutions théoriques connues, mais d'envisager le calcul en condition normale d'utilisation (ouvrage-type). L'organisation des concours de prévisions répond en grande partie à cet objectif, mais les facteurs d'incertitude évoqués précédemment viennent compliquer et rendre très coûteux le processus de validation des modèles et de formation des utilisateurs par l'exemple. Pour répondre à cette question de "rentabilité ", des cas-tests de type semi-industriel ont été proposés avec le double objectif d'être à la fois un outil de validation et un outil d'apprentissage (qualification de l'utilisateur). Il s'agit ainsi

- soit de comparer des codes entre eux à l'aide d'un cas-test d'ouvrage défini de manière à s'affranchir des problèmes liés à la précision de la technique numérique (toutes les données sont fixées : maillage, comportement des matériaux, conditions aux limites, chargements, etc.). Ces tests peuvent également aider au choix d'un logiciel dans un appel d'offres ;

- soit d'apprécier l'emploi d'un code par différents utilisateurs pour traiter un même problème de géotechnique tout en laissant libres les conditions de calcul : maillage (choix des éléments et du raffinement, etc.); choix des incréments pour une résolution en comportement non linéaire ; etc.

H. Schweiger est le premier, semble-t-il, à avoir proposé et publié des cas-tests de ce type à partir de 1991. L'enjeu est toutefois plus large, car de leur élaboration dépendent aussi des propositions visant d'une part à la formation par l'exemple des jeunes ingénieurs à la modélisation et, d'autre part, à l'organisation de tests d'aptitude ou de vérification de compétences en matière de modélisation. La formation initiale et continue des utilisateurs est un enjeu d'importance pour la réalisation de bons modèles et pour la qualité des calculs.

\section{Conclusions}

Même si tout ouvrage de géotechnique est un " exemplaire unique " de par ses structures et son environnement, la modélisation de l'ouvrage ne peut être unique tant les hypothèses sont nombreuses pour l'élaborer. Chaque ingénieur a une certaine conception du fonctionnement d'un ouvrage et il construira son modèle en fonction de ses connaissances. Pour ces deux raisons, la validation d'un modèle reste une affaire ponctuelle et somme toute relative.

Néanmoins, la publication d'études de synthèse sur des expérimentations et des modélisations d'ouvrages-types a permis de proposer des critères objectifs pour la validation et la justification des modèles d'éléments finis. La question essentielle 
n'est pas de connaître le degré d'exactitude des valeurs numériques, mais d'estimer le réalisme de la modélisation élaborée.

Par ailleurs comme, malgré tous les tests effectués, on ne peut garantir l'absence d'erreur dans un logiciel, les résultats de calcul par éléments finis doivent être examinés avec rigueur et soumis à l'avis de géotechniciens avertis.

Les tests de validation sont essentiels mais ils ne sont donc pas suffisants. Des méthodes d'Assurance-Qualité dans le développement, la maintenance et l'utilisation du logiciel doivent venir les complèter. C'est dans la combinaison de ces deux garanties que résident la meilleure preuve de la fiabilité du logiciel et donc les arguments suffisants pour convaincre de la qualité des calculs les responsables de bureaux d'études et de contrôle.

\section{Bibliographie}

[AAL 98] Aalto A., Rekonen R., LoJANDer M. (1998) The calculations on haarajoki test embankment with the finite element program PLAXIS 6.31. Applications of numerical methods to geotechnical problems, NUMGE98, Cividini (ed), Springer, pp. 37-46.

[CAM 95] Cambou B., Favre J.-L., Lanier J., Shahrour I., Shao J.-F. (1995) Validation des codes de calcul. Mécanique des géomatériaux, Darve, Hicher et Reynouard (eds), Hermès, pp. 445-562.

[CIG 94] CIGB (1994) Logiciels de calcul des barrages. Validation. Réflexions et propositions. Commission Internationale des Grands Barrages, Bulletin 94, 128 pages.

[DEB 89] De BruYn D., Aubry D., Rousset G. (1989) Comparison of rheological models in view of predicting the behaviour of a deep clay host rock during the construction of a radwaste repository. Numerical Models in Geomechanics, NUMOG III, Pande et Pietruszczak (eds), Balkema, pp. 724-731.

[GAU 98] Gaudel M.C., Marre B., Schlienger F. (1998) Modèles de développement du logiciel. Revue de l'Electricite et de l'Electronique, $\mathrm{n}^{\circ} 6$, juin 1998, pp. 34-40.

[GIB 94] GibbenS R., BRIAUD J.-L. (1994) Data and prediction request for the spread footing prediction event. Settlement '94 - ASCE Specialty Conference, Texas A\&M University, pp. 12-85.

[LAM 73] Lambe T.W. (1973) «Predicition in soil engineering " géotechnique, vol. 23, pp. 149-202

[LEP 90] LEPIDAS I., MAGNAN J.-P. (1990) Fluage et consolidation des sols argileux : modélisation numérique. Rapport de recherche des $L P C, \mathrm{n}^{\circ} 157$, Laboratoire Central des Ponts et Chaussées, 170 pages.

[MAG 86] MAGNAN J.-P. (1986) Modélisation numérique du comportement des argiles molles naturelles. Rapport de recherche des $L P C, \mathrm{n}^{\circ} 141$, Laboratoire Central des Ponts et Chaussées, 255 pages.

[MAG 92] MAGNAN J.-P. (1992) Les remblais d'essai sur argiles molles de Muar Flats (Malaisie). Etudes et Recherches des Laboratoires des Ponts et Chaussées, série Géotechnique, GT 50, 65 pages. 
[MAG 97] Magnan J.-P., Mestat Ph., LeCa E., Humbert P. (1997) Expérimentations et modélisations au LCPC : 30 ans de comparaison entre comportements observés et calculés. Colloque AUGC - EC'97, Strasbourg, 22-23 mai 1997, vol. 1, pp. 55-62.

[MES 99] Mestat Ph., Humbert P., Dubouchet A. (1999) Type d'erreurs et de vérifications dans les calculs par éléments finis. Colloque AUGC-AFGC "Quels systèmes qualité pour la modélisation par éléments finis? ", 6 et 7 mai, Poitiers.

[PIP 88] PIPER D., KNOWLES N.C. (1988) Some computational experiences of a geomechanical benchmark in rock salt. Numerical Methods in Geomechanics, Swoboda (ed), Balkema, pp. 189-193.

[PRA 95] Prat M., Bisch Ph., Mestat Ph., Millard A., Puaudier-Cabot G. (1995) La modélisation des ouvrages, Éditions Hermès, 780 pages.

[PRA 97] Prat M., Bisch Ph., Mestat Ph., Millard A., Puaudier-Cabot G. (1997) Calcul des ouvrages généraux de construction, Editions Hermès, 770 pages.

[PRI 91] PRINTZ J. (1991) Le génie logiciel. Collection Que sais-je ? Presses Universitaires de France.

[SCH 91] SCHWEIGER H. (1991) Some results from benchmark tests for geotechnical engineering. Numerical Models in Geomechanics - NUMOG V, Pande et Pietruszczak (eds), Balkema, pp. 675-680.

[SCH 98] SCHWEIGER H. (1998) Results from two geotechnical benchmark problems. Applications of numerical methods to geotechnical problems, NUMGE98, Cividini (ed), Springer, pp. 645-654.

[TAR 97] TARDIEU B., OzANAM O. (1997) Le modèle accompagnateur de projet. Revue Française de Génie Civil, vol. 1, numéro hors série, 160 pages.

[WOL 94] von Wolffersdorff P.A. (1994), "Results of the field test and evaluation of the predictions ans subsequent calculations" Workshop Sheet Pile Test Karlsruhe, Delft University, Holland, October 1994. 